ISSN 2075-4701

www.mdpi.com/journal/metals/

Editorial

\title{
Manganese-based Permanent Magnets
}

\section{Ian Baker}

Thayer School of Engineering, Dartmouth College, 14 Engineering Drive, Hanover, NH 03755-8000, USA; E-Mail: ian.baker@dartmouth.edu; Tel.: +1-603-646-2184; Fax: +1-603-646-3856

Received: 10 August 2015 / Accepted: 10 August 2015 / Published: 11 August 2015

There is a significant gap between the energy product, $\mathrm{BH}$, where $\mathrm{B}$ is the magnetic flux density and $\mathrm{H}$ is the magnetic field strength, of both the traditional ferrite and AlNiCo permanent magnets of less than 10 MGOe and that of the rare earth magnets of greater than 30 MGOe. This is a gap that Mn-based magnets could potentially, inexpensively, fill. This Special Issue presents work on the development of both types of manganese permanent magnets. Some of the challenges involved in the development of these magnets include improving the compounds' energy product, increasing the thermal stability of these metastable compounds, and producing them in quantity as a bulk material.

The aim of this Special Issue is to address these challenges from both experimental and theoretical points of view. Four of the papers are focused on $\mathrm{MnAl}$ and $\mathrm{MnBi}$, which exhibit a $\mathrm{L} 10$ tetragonal structure. Three of the papers are concerned with processing of MnAl either by rapid solidification to ribbons followed by annealing [1], or by mechanical milling of rapidly-solidified powders followed by either simply annealing [2] or by warm consolidation to bulk material using equal channel angular extrusion [3]. In the paper by Park et al. [4], the magnetic moment, magnetocrystalline anisotropy energy, Curie temperature, and electronic structure of $\mathrm{MnBi}$ have been calculated. Interestingly, their results suggest that doping the interstitial sites of $\mathrm{MnBi}$ with an interstitial can increase the saturation magnetization. The final paper by Sugihara et al. [5] focuses on the behavior of $\mathrm{Mn}_{3} \mathrm{Ge}$ thin films, which exhibit a tetragonal Heusler-like $\mathrm{D}_{22}$ crystal structure.

\section{References}

1. Jiménez-Villacorta, F.; Marion, J.L.; Oldham, J.T.; Daniil, M.; Willard, M.A.; Lewis, L.H.; Magnetism-Structure Correlations during the $\varepsilon \rightarrow \tau$ Transformation in Rapidly-Solidified MnAl Nanostructured Alloys. Metals 2014, 4, 8-19.

2. Lucis, M.J.; Prost, T.E.; Jiang, X.; Wang, M.; Shield, J.E. Phase Transitions in Mechanically Milled Mn-Al-C Permanent Magnets. Metals 2014, 4, 130-140.

3. Chaturvedi, A.; Yaqub, R.; Baker, I. Microstructure and Magnetic Properties of Bulk Nanocrystalline MnA1. Metals 2014, 4, 20-27. 
4. Park, J.; Hong, Y.-K.; Lee, J.; Lee, W.; Kim, S.-G.; Choi, C.-J. Electronic Structure and Maximum Energy Product of MnBi. Metals 2014, 4, 455-464.

5. Sugihara, A.; Suzuki, K.; Miyazaki, T.; Mizukami, S. Epitaxial Growth of Hard Ferrimagnetic Mn3Ge Film on Rhodium Buffer Layer. Metals 2015, 5, 910-919.

(C) 2015 by the authors; licensee MDPI, Basel, Switzerland. This article is an open access article distributed under the terms and conditions of the Creative Commons Attribution license (http://creativecommons.org/licenses/by/4.0/). 